\title{
An Analytical Procedure by the Inductively Coupled Plasma Mass Spectrometry (ICP-MS) for the Quantitation of Total Phosphorus Content on the Surface of the Manufacturing Equipment for Oligonucleotides
}

\author{
Daisuke Furukawa, Masami Nakai, Masato Kazusaki* \\ Quality Assurance Office Health \& Crop Science Sector, Sumitomo Chemical Co., Ltd., Osaka, Japan
}

Email address:

kazusakim@sc.sumitomo-chem.co.jp (M. Kazusaki)

${ }^{*}$ Corresponding author

To cite this article:

Daisuke Furukawa, Masami Nakai, Masato Kazusaki. An Analytical Procedure by the Inductively Coupled Plasma Mass Spectrometry (ICP-MS) for the Quantitation of Total Phosphorus Content on the Surface of the Manufacturing Equipment for Oligonucleotides. Science Journal of Analytical Chemistry. Vol. 8, No. 1, 2020, pp. 1-4. doi: 10.11648/j.sjac.20200801.11

Received: December 25, 2019; Accepted: January 7, 2020; Published: January 30, 2020

\begin{abstract}
An analytical procedure by the inductively coupled plasma mass spectrometry (ICP-MS) is provided to quantitate the remaining phosphorus amount on the surface of the manufacturing equipment for the oligonucleotide active pharmaceutical ingredient (API). In this analytical procedure, the sample solutions obtained from the rinse sampling and direct surface sampling methods are directly injected into the ICP-MS equipment without the inspissation of the extraction liquid. The limit of quantitation for phosphorous was $0.02 \mu \mathrm{g} / \mathrm{mL}$. Quantitation of phosphorous was achieved over a linear range of 0.02 to 0.8 $\mu \mathrm{g} / \mathrm{mL}$. Analytical validation was carried out to prove that this analytical procedure is appropriate and effective to monitor the cleanliness of the manufacturing equipment after the oligonucleotide API manufacturing and the following cleaning of the equipment. Calibration curve originally passed through the origin with correlation coefficient of 0.999 . The precision through the rinse sampling method was within $\pm 10 \%$. The satisfactory accuracy for the rinse sampling method was proven from the assessment of linearity and precision. The precision and accuracy of the analytical procedure were both within $\pm 10 \%$ for the direct surface sampling method. The described analytical procedure by ICP-MS following the two types of the sampling procedures can easily be applied to the routine analysis for the verification of the cleanliness of the manufacturing equipment for oligonucleotide API.
\end{abstract}

Keywords: ICP-MS, Oligonucleotide, Validation, Sampling, Manufacture, Cleanliness

\section{Introduction}

Synthetic oligonucleotide active pharmaceutical ingredients (API) presents potential towards therapeutics to large number of patients with unmet medical remedies [1-11]. The quantitation of the total amount of the synthesized oligonucleotide API on the surface of the manufacturing equipment is a prerequisite to manufacture the API under the GMP principle [12]. The reminding oligonucleotide API on the manufacturing equipment surface is taken by the direct surface sampling method or the rinse sampling method.

Because of ability and convenience, ultraviolet - visible absorbance spectroscopic procedure is used to quantitate the residual oligonucleotide amount on the manufacturing equipment in our facility. However, the limitation of this procedure is a lack of adequate specificity to determine the target compounds. The above-mentioned procedures would also require the time-consuming process for inspissation of the extraction liquid due to relatively poor limit of quantitation. So, we developed the new quantitation procedure to assess the cleanliness of the manufacturing equipment for oligonucleotides.

The inductively coupled plasma mass spectrometry (ICP-MS) has been employed for multi-elemental analysis and the independent measurement of stable isotopes [13-15]. ICP-MS was proved to possess an effective combination of accuracy, precision. Many elements were quantitated with 
good detection limits [16]. The high specificity of ICP-MS enables ultra-trace quantitation of phosphorous in the sample solution. So, we took a particular note on the quantitation of phosphorous to assess the oligonucleotide content on the manufacturing equipment, because phosphorous is essential to construct the RNA molecules. The oligonucleotide content is easily calculated from the phosphorous content determined by ICP-MS. To the best of our knowledge, we are the first group to report the validated analytical procedure by ICP-MS to quantitate the remaining oligonucleotide API on the manufacturing equipment through the quantitation of phosphorous. The aim of this work was to develop an analytical procedure with ICP-MS to be implemented in the routine examination of the cleanliness of the manufacturing equipment employed for the oligonucleotide manufacturing under the GMP principle.

\section{Experimental}

\subsection{Materials}

Phosphorous standard solution $1000 \mathrm{mg} / \mathrm{L}$ was purchased from FUJIFILM Wako Pure Chemical Corporation (Osaka, Japan). In all cases, water was purified by deionization with minimum conductivity value of $18.0 \mathrm{~m} \Omega / \mathrm{cm}$. Tuning Solution for ICP-MS was obtained from Agilent Technologies Japan, Ltd., (Tokyo, Japan). Oligonucleotide active pharmaceutical ingredient (API) was from Sumitomo Chemical Co., Ltd., (Tokyo, Japan).

\subsection{Equipment}

ICP-MS, Agilent model 7700x, was employed in this study. This ICP-MS has been housed in a thermostatically controlled room at $20^{\circ} \mathrm{C}$. The major isotope of phosphorous was monitored at $\mathrm{m} / \mathrm{z}$ 31. Quantitation was based on the average of the three values of count per second against a calibration curve.

\subsection{Preparation of the Standards, Matrices for the Validation Study and Quality Control Samples}

The standard solutions for linearity were also prepared from the phosphorous standard solution, covering the concentration range of 0.02 to $0.8 \mu \mathrm{g} / \mathrm{mL}$ with or without the oligonucleotide API.

Validation matrices of the rinse sampling method for accuracy and precision were the oligonucleotide API solutions in the concentration range of 0.2 to $10 \mu \mathrm{g} / \mathrm{mL}$ corresponding to 0.02 to $0.8 \mu \mathrm{g} / \mathrm{mL}$ of phosphorous.

Validation matrices of the direct surface sampling solution for accuracy and precision were the oligonucleotide API solutions at the concentration levels of $0.2 \mu \mathrm{g} / \mathrm{mL}$ and 10 $\mu \mathrm{g} / \mathrm{mL}$ corresponding to $0.02 \mu \mathrm{g} / \mathrm{mL}$ and $0.8 \mu \mathrm{g} / \mathrm{mL}$ of phosphorous. Calibration standards for the quantitation of phosphorous in the quality control solutions for the cleanliness verification were freshly prepared by the dilution of the phosphorous standard solution, resulting in the concentration range of 0.2 to $0.8 \mu \mathrm{g} / \mathrm{mL}$.
After the cleaning procedure of the manufacturing equipment for oligonucleotide API as the GMP operation, the surface of equipment was swabbed with the fabric. Then, the fabrics were immersed into the water to extract the oligonucleotide API adsorbed on the fabrics from the equipment surface. For the equipment with difficulty in swabbing the equipment surface, inside of the equipment was rinsed with water. The extract liquid obtained from the fabrics and rinse solution were both employed as the quality control samples.

\section{Results and Discussion}

The analytical procedure for the cleaning verification with ICP-MS was validated for the following validation characteristics according to the general guidelines [17, 18].

Limit of quantitation (LOQ) was evaluated as the minimum concentration at which the relative standard deviation obtained from the multiple injection of the phosphorous standard was equal to or less than $10 \%$. From this investigation, the LOQ was set to be $0.02 \mu \mathrm{g} / \mathrm{mL}$. From the definition of the limit of detection (LOD), LOD was established $0.007 \mu \mathrm{g} / \mathrm{mL}$.

The linearity was investigated by making injections of the standard solution containing phosphorous, and assessed by linear regression analysis with the relationship between the phosphorous concentration and response in count per second. Two calibration curves were constructed with the standard solutions containing phosphorous at five concentration levels in the range of $0.02-0.8 \mu \mathrm{g} / \mathrm{mL}$ covering the analytical results from the previous cleanliness verification conducted at the end of the oligonucleotide API manufacturing campaign previously conducted by Sumitomo Chemical Co., Ltd. One standard solution is prepared by the dilution of the Phosphorous standard solution $1000 \mathrm{mg} / \mathrm{L}$ purchased from FUJIFILM Wako Pure Chemical Corporation, and another by dissolving oligonucleotide API. Calibration curve was constructed by plotting response against the phosphorous concentration in the standard solutions (Figure 1), and the two calibration curves were evaluated by regression analysis.

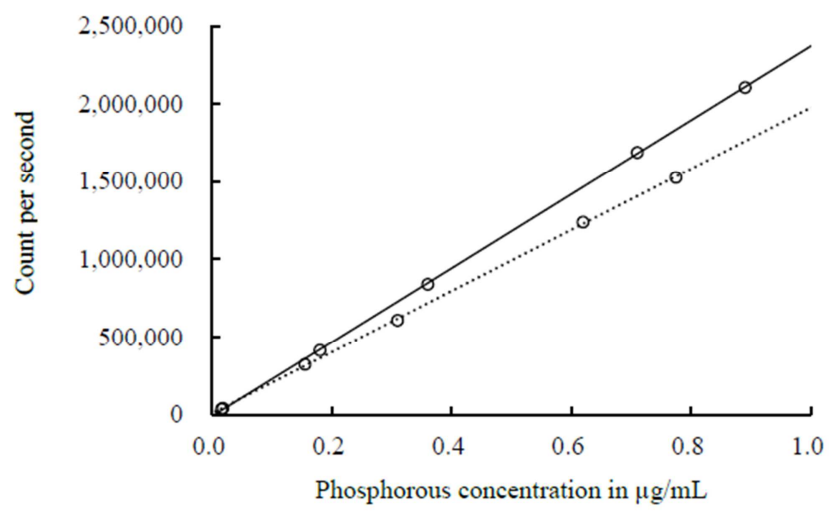

Figure 1. Calibration curve for phosphorous. Solid line; phosphorous from the standard solution; dotted line, phosphorous from the oligonucleotide API.

Correlation coefficients of these two calibration curves are 
both 0.999 . Zero point of $y$-axis was found in the interval of $95 \%$ confidence level of intercept of $y$-axis for both two calibration curves, indicating that these two calibration curves generated in the presence or absence of the oligonucleotide API passed through the origin. This fact suggests that the spectral interference for the measurement of phosphorous by ICP-MS is negligible in this analytical procedure. Differences between the observed values and the calculated values from the corresponding regression line are scattered naturally at the corresponding concentration on the $x$-axis. Linearity of this calibration curve is evaluated according to the criteria described in the USP. Acceptance criteria for linearity is equal to or more than 0.99 for the impurities quantitated by the ICP-MS according to the USP. Our result satisfies the acceptance criteria described in the USP. Ratio of the two slopes for the calibration curves generated with and without the oligonucleotide API is 1.2, suggesting the correction by the factor of 1.2 is essential due to the non-spectral interference to evaluate the actual amount of phosphorous on the surface for manufacturing of oligonucleotide API. Based on these results, the calibration curve with the correction factor of 1.2 was adopted for the quantitation of phosphorous in the quality samples for the verification of cleanliness of the manufacture equipment for the oligonucleotide API.

For the rinse sampling method, precision was evaluated as the standard deviation from regression analysis of the calibration curve. Point estimation of the standard deviation of the calibration curve showing the relationship between the response and the phosphorous concentration from the oligonucleotide API (dotted line in Figure 1) was 5.6\%. This value satisfies the acceptance criteria for precision described in the USP. Acceptance criteria for precision is equal to or less than $20 \%$ for the impurities quantitated by the ICP-MS according to the USP. Our result satisfies the acceptance criteria described in the USP. In addition, the $90 \%$ confidence interval was the range of $3.4 \%$ to $16.2 \%$. Accuracy was estimated to be acceptable from the linearity and precision of the rinse method.

Accuracy and precision for the direct surface sampling method were assessed by the quantitation of the phosphorous from the oligonucleotide API spiked onto the metal plates composing the synthetic equipment for the API. An aliquots of oligonucleotide API in the water was spiked onto the three kinds of the metal plates. Spiked amount of phosphorous was $0.9 \mu \mathrm{g}$, respectively. After drying to mimic the actual status of the equipment for oligonucleotide manufacture, the surface was swabbed by the fabric. The oligonucleotide adsorbed on the fabric was extracted into the water and analyzed by ICP-MS. Accuracy and precision were expressed as the average and the standard deviation of the difference between the observed amount and the spiked amount. Point estimation of the average of the difference between the observed amount and the spiked amount was $0.7 \%$. This value satisfies the acceptance criteria for accuracy described in the USP. According to the USP, acceptance criteria for recovery is in the range of $70 \%$ to
$150 \%$ corresponding to $-30 \%$ to $50 \%$ for the impurities quantitated by the ICP-MS. Our result satisfies the acceptance criteria described in the USP. Zero in the y-axis was in the range of the $95 \%$ confidence interval of $-2.3 \%$ to $3.7 \%$, suggesting the negligible systematic error from the surface sampling method followed by the ICM-MS analysis Point estimation of the standard deviation of the difference between the observed amount and the spiked amount was $1.7 \%$. This value satisfies the acceptance criteria for precision described in the USP (acceptance criteria for precision is equal to or less than $20 \%$ for the impurities quantitated by the ICP-MS). In addition, the $90 \%$ confidence interval was the range of $1.2 \%$ to $3.3 \%$.

After the cleaning of the manufacturing equipment, the ICP-MS analytical procedure following the sampling method was applied to the actual manufacturing equipment for oligonucleotide API. Any amount quantitated as the residual oligonucleotide API on the each equipment was less than the LOQ level. In case of detection the trace amount of phosphorous was less than LOQ, the residual amount was estimated to remain at the level of LOQ. Total amount of residual oligonucleotide API were calculated from the swabbed area to the total surface of the equipment or the relationship of the volume of the rinse solution to the inside volume of equipment. Finally, the estimated residual amount was converted to the ratio of the contamination level to the subsequent manufacturing oligonucleotide based on the manufacturing scale. The estimated residual amounts on the manufacturing equipment are shown in Table 1.

Table 1. Residual amount of oligonucleotide on the manufacturing equipment (ppm).

\begin{tabular}{ll}
\hline Manufacturing process & $\begin{array}{l}\text { Amount of the oligonucleotide API on } \\
\text { the equipment }\end{array}$ \\
\hline Solid phase synthesis & $<11.2$ \\
Annealing & $<5.2$ \\
Freeze drying & $<4.9$ \\
Total amount & $<21.3$ \\
\hline
\end{tabular}

The maximum amount of the residual oligonucleotide API was estimated to be $21.3 \mathrm{ppm}$ to the amount of the oligonucleotide API in the following manufacture. This residual level is toxicologically acceptable to assure the quality of the oligonucleotide API in the subsequent manufacturing campaign.

In contrast to other analytical technique, ICP-MS quantitate the target elemental without the molecular specific properties lost in the plasma ion source. Recently, some state-of-the-art separation techniques such as capillary electrophoresis, HPLC, gel electrophoresis are applied to selectively quantitate the target compounds with ICP-MS [19-22]. If these techniques are applied to the oligonucleotide residue on the manufacturing equipment, the full-length oligonucleotide are separated from the shortmers that are the process impurities generating from the manufacture of the oligonucleotide API by the deletion of a nucleotide or more from the full-length oligonucleotide API. If the oligonucleotide APIs are administered into the living system, 
they are immediately decomposed by the nuclease. In this case, from the view of safety it is not essential to separate the full-length oligonucleotide from shortmers, because these compounds are decomposed immediately into shorter oligonucleotides up to the corresponding nucleases in the living system. It is the reason that we quantitate all the amount of phosphorus on the surface of the manufacturing equipment as the target compound of the full-length oligonucleotide and shortmers.

\section{Conclusion}

We have developed the validated ICP-MS analytical procedure to quantify the total phosphorous amount on the manufacturing equipment surface for oligonucleotide API. Our analytical procedure does not require the time-consuming sample preparation process. This procedure was successively applied to verify the cleanliness of the equipment for the oligonucleotide API synthesis.

Acceptable limit for the residual amount of all the oligonucleotides on the manufacturing equipment was set forth according to the threshold of the toxicological concern [23]. Residual amount of the oligonucleotides on the actual manufacturing equipment should be under the acceptance limit considered from the safety aspects for the patients. This procedure should be carried out at our factory to verify the cleanliness of the equipment.

\section{References}

[1] S. Crooke, Antisense Nucleic Acid Drug Dev. 8 (2) (1998) 133.

[2] S. Crooke, Vitravene - another piece in the mosaic, Antisense Nucleic Acid Drug Dev. 8 (4) (1998) (vii-viii).

[3] T. Abe, T. Mizuta, T. Hatta, N. Miyano-Kurosaki, M. Fujiwara, K. Takai, S. Shigeta, T. Yokota, H. Takaku, Antisense therapy of influenza, Eur. J. Pharm. Sci. 13 (2001) 61-69.

[4] N. Dias, C. A. Stein, Antisense oligonucleotides: basic concepts and mechanisms, Mol. Cancer Ther. 1 (2002) 347355 .

[5] N. M. Dean, F. Bennett, Antisense oligonucleotide-based therapeutics for cancer, Oncogene 22 (2003) 9087-9096.

[6] C. Mello, D. Conte, Revealing the world of RNA interference, Nature 431 (2004) 338-342.

[7] D. P. Bartel, MicroRNAs: genomics, biogenesis, mechanism, and function, Cell 116 (2004) 281-297.

[8] S. Doggrell, Expert Opin. Pharmacother. 6 (8) (2005) 1421.

[9] S. Doggrell, Pegaptanib: the first antiangiogenic agent approved for neovascular macular degeneration, Expert Opin. Pharmacother. 6 (8) (2005) 1421-1423.
[10] S. T. Crooke, An overview of progress in antisense therapeutics, Antisense Nucleic Acid Drug Dev. 8 (2) (2009) 115-122.

[11] A. C. McGinnis, B. Chen, M. G. Bartlett, Chromatographic methods for the determination of therapeutic Oligonucleotides, J. Chromatogr. B 883 (2012) 76-94.

[12] International Council for Harmonisation of Technical Requirements for Pharmaceuticals for Human Use, GOOD MANUFACTURING PRACTICE GUIDE FOR ACTIVE PHARMACEUTICAL INGREDIENTS Q7, 2000. https://www.pmda.go.jp/files/00.026709.pdf.

[13] C Diane Beauchemin, Inductively Coupled Plasma Mass spectroscopy, Anal. Chem., 2010, 82, 12, 4786-4810.

[14] D R. S. Houk., Mass spectrometry of inductively coupled plasmas, Anal. Chem., 1986, 58, 1, 97A-105A.

[15] E Scott C Wilschefski, Matthew R Baxter, Inductively Coupled Plasma Mass Spectrometry: Introduction to Analytical Aspects, Clin Biochem Rev., 2019 Aug; 40 (3): 115-133.

[16] Jeffrey R. Bacon, Jeffrey S. Crain, Luc Van Vaeck and John G. Williams, Atomic mass spectrometry, Journal of Analytical Atomic Spectrometry, 14 (10), 1633-1659.

[17] Pharmacopoeia of the United States of America (USP).

[18] International Council for Harmonisation of Technical Requirements for Pharmaceuticals for Human Use, VALIDATION OF ANALYTICAL PROCEDURES: TEXT AND METHODOLOGY Q2 (R1), 2005. https://www.pmda.go.jp/files/00.026867.pdf.

[19] D. Pro" frock, P. Leonhard, A. Prange. "Determination of phosphorus in phosphorylated deoxyribonucleotides using capillary electrophoresis and high performance liquid chromatography hyphenated to inductively coupled plasma mass spectrometry with an octopole reaction cell". J. Anal. At. Spectrom. 2003. 18 (7): 708-713.

[20] M. Edler, N. Jakubowski, M. Linscheid. "Quantitative determination of melphalan DNA adducts using HPLC inductively coupled mass spectrometry". J. Mass. Spectrom. 2006. 41 (4): 507-516.

[21] D. G. Sar, L. Aguado, M. M. Bayon, M. A. Comendador, E. B. Gonzalez, A. Sanz-Medel, L. M. Sierra. "Relationships between cisplatin-induced adducts and DNA strandbreaks, mutation and recombination in vivo in somatic cells of Drosophila melanogaster, under different conditions of nucleotide excision repair". Mutation Research-Genetic Toxicology and Environmental Mutagenesis. 2012. 741 (1-2): 81-88.

[22] L. L. Fernandez, M. Montes-Bayon, E. B. Gonzalez, L. M. Sierra, A. Sanz-Medel, J. Bettmer. "Initial studies on quantitative DNA induced oxidation by gel electrophoresis (GE)-ICP-MS". J. Anal. At. Spectrom. 2011.26 (1): 195-200.

[23] International Council for Harmonisation of Technical Requirements for Pharmaceuticals for Human Use, ASSESSMENT AND CONTROL OF DNA REACTIVE (MUTAGENIC) IMPURITIES IN PHARMACEUTICALS TO LIMIT POTENTIAL CARCINOGENIC RISK M7 (R1), 2017. http://www.pmda.go.jp/files/000218750.pdf. 